objective was to determine if delayed cyclosporine treatment was still effective in protecting asphyxiated piglets. We hypothesize that both early and delayed treatment with cyclosporine A would improve cardiac recovery during resuscitation of asphyxiated newborn piglets.

Methods Thirty piglets (1-4 days-old) were instrumented for continuous monitoring. After stabilization, normocapnic alveolar hypoxia (10-15\% oxygen) was instituted for $2 \mathrm{~h}$ followed by reoxygenation for $6 \mathrm{~h}$. Piglets were block-randomized to receive either early (5 min of reoxygenation) or delayed (120 min reoxygenation) intravenous bolus of cyclosporine $(10-\mathrm{mg} / \mathrm{kg})$ or saline (control) at identical times during reoxygenation ( $n=8 /$ group). Myocardial and intestinal lactate concentrations as well as histological examinations were determined.

Results Hypoxic piglets had cardiogenic shock (cardiac output $52 \pm 1 \%$ of baseline), hypotension and acidosis. Although both early and delayed cyclosporine treatment improved cardiac output $(\mathrm{P}<0.05$ vs. controls), only early cyclosporine treatment improved stroke volume and systemic oxygen delivery ( $p<0.05 \mathrm{vs}$. controls). Left ventricle and intestinal lactate were higher in controls than in both cyclosporine-treated groups. Early, but not delayed, cyclosporine treatment also attenuated intestinal injury compared to controls $(\mathrm{P}<0.05)$.

Conclusion This study demonstrates that both early and delayed cyclosporine treatment during resuscitation improves cardiac recovery in asphyxiated newborn piglets. However, early treatment with cyclosporine may offer superior cardioprotection and attenuates $\mathrm{H}-\mathrm{R}$ intestinal injury.

\section{THE CARDIO-PROTECTIVE EFFECTS OF DOXYCYCLINE IN A SWINE MODEL OF NEONATAL ASPHYXIA}

doi:10.1136/archdischild-2012-302724.0317

1J LaBossiere, 'JS Pelletier, 'D Bigam, 1,2PY Cheung. 'Surgery; ${ }^{2}$ Pediatrics and Pharmacology, University of Alberta, Edmonton, AB, Canada

Background Myocardial reoxygenation injury following asphyxia in neonates is common. Matrix metalloproteinase-2 activation is associated with myocardial ischemic-reperfusion injury and stunning. Previous in vitro, ex vivo, and small animal studies have demonstrated the cardio-protective qualities of doxycycline, a known inhibitor of matrix metalloproteinase-2, however, large animal models demonstrating this effect are lacking. We hypothesized that doxycycline would improve cardiac recovery and systemic hemodynamics in asphyxiated newborn piglets.

Methods Piglets (1-5 days old) were acutely instrumented for continuous monitoring of heart rate, cardiac output [CO], mean systemic and pulmonary arterial pressures (SAP and PAP, respectively). After stabilization, $2 \mathrm{hrs}$ of normocapnic alveolar hypoxia (10-15\% oxygen) was induced followed by 4 hrs of normoxic reoxygenation ( $21 \%$ oxygen). Piglets were blindly randomized to receive either normal saline or doxycycline $(3,10$, or $30 \mathrm{mg} / \mathrm{kg}$ ) intravenously 5 minutes into reoxygenation ( $\mathrm{n}=7 /$ group). Sham-operated piglets $(\mathrm{n}=5)$ received no hypoxia-reoxygenation.

Results Asphyxiated piglets demonstrated acidosis ( $\mathrm{pH}=7.04 \pm$ $[\mathrm{SD}] 0.08)$, hypotension $(\mathrm{SAP}=31 \pm 3 \mathrm{mmHg}$ ), and cardiac dysfunction $(\mathrm{CO}=58 \pm 8 \%$ of normoxic baseline). Doxycycline had dose-related improvements in $\mathrm{CO}$ and stroke volume $(30 \mathrm{mg} / \mathrm{kg}: 86 \pm 8 \%$ and $79 \pm 15 \%$ of normoxic baseline vs. $65 \pm 7 \%$ and $50 \pm 13 \%$ in controls, respectively [both $\mathrm{p}<0.05]$ ), with no significant change in heart rate compared to controls. Furthermore, SAP was higher and PAP/SAP ratio was lower than those of controls $(p<0.05)$, with no difference in PAP.

Conclusions In an established swine model of neonatal hypoxiareoxygenation, post-resuscitation administration of intravenous doxycycline improves cardiac recovery with beneficial hemodynamic effects in systemic and pulmonary circulations.

Funded by Canadian Institutes of Health Research (CIHR).

\section{CAN EARLY B-TYPE NATRIURETIC PEPTIDE ASSAYS PREDICT SYMPTOMATIC PATENT DUCTUS ARTERIOSUS IN EXTREMELY LOW BIRTH WEIGHT INFANTS?}

doi:10.1136/archdischild-2012-302724.0318

${ }^{1} \mathrm{JH}$ Lee, ${ }^{2} \mathrm{JH}$ Shin, ${ }^{3} \mathrm{KH}$ Park, ${ }^{2} \mathrm{YJ}$ Rhie, ${ }^{1} \mathrm{MS}$ Park, ${ }^{2} \mathrm{BM}$ Choi. 'Department of Pediatrics, Ajou University School of Medicine, Suwon; '2Departement of Pediatrics, Korea University College of Medicine, Ansan-Si; ${ }^{3}$ Department of Pediatrics, CHA Gangnam Medical Center, Seoul, Republic of Korea

Background and amis Optimal management of a patent ductus arteriosus (PDA) is important for improving the clinical outcomes of extremely low birth weight (ELBW) infants. Although it is reported that prophylactic cyclooxygenase inhibitors result in favorable immediate outcomes, not only serious side effect such as nephrotoxicity but also unnecessarily drug exposure without benefit are inevitable. To investigate the predictability of B-type natriuretic peptide (BNP) for early targeted treatment of hemodynamically significant PDA (hsPDA) in ELBW infants.

Methods 73 ELBW infants that underwent echocardiographic evaluation and plasma BNP measurement after birth were enrolled. 31 infants developed hsPDA (HsPDA group) and 42 infants didn't develop hsPDA (nPDA group).

Results BNP levels of HsPDA group were significantly higher than those of nPDA group at 24 hours of age (921[318-2133] vs. 152 [91-450], pg/mL) but not different at 12 hours of age. BNP levels at 24 hours of age were significantly correlated with the magnitudes of the ductal shunt but not significant at 12 hours of age. The area under the receiver operator characteristic curve of BNP levels for prediction of hsPDA at 12 and 24 hours of age was 0.584 and 0.830 , respectively. At the cutoff BNP level of $200 \mathrm{pg} / \mathrm{mL}$ and $900 \mathrm{pg} / \mathrm{mL}$ at 24 hours of age, the sensitivity was $83.9 \%$ and $54.8 \%$ and the specificity was $61.9 \%$ and $95.2 \%$, respectively.

Conclusions BNP levels at 24 hours of age can be used as a guide for early targeted treatment of hsPDA and avoid the unnecessary use of cyclooxygenase inhibitors in ELBW infants.

\section{THE EFFECT OF MILRINONE INFUSION ON CEREBRAL PERFUSION IN NEONATES WITH CONGENITAL HEART DISEASE PRIOR TO CARDIAC SURGERY}

doi:10.1136/archdischild-2012-302724.0319

1,2MO Bianchi, 'PY Cheung, 'E Phillipos, ${ }^{2} \mathrm{~A}$ Aranha-Netto, ${ }^{1} \mathrm{C}$ Joynt. 'Pediatrics, University of Alberta, Edmonton, AB, Canada; ${ }^{2}$ Pediatrics, UNICAMP, Campinas, Brazil

Background Milrinone has been increasingly used in the postoperative care of neonates with congenital heart disease (CHD) for its inotropic and vasodilatation properties. When used as a preoperative cardiovascular supportive agent, cerebral hemodynamic effects of milrinone have not been studied.

Methods From June 2008 to July 2010, 18 neonates with CHD and treated with milrinone before cardiac surgery were prospectively enrolled. Milrinone $(0.75 \mathrm{mcg} / \mathrm{kg} / \mathrm{min})$ was given according to institutional guidelines and neonates $(n=1)$ requiring additional vasoactive agents were excluded. Using Doppler studies, cardiac output (CO), flow velocity $(\mathrm{Vm})$ and Resistive Index (RI) for anterior and middle cerebral arteries were assessed and analyzed blindly at specific time-points after milrinone administration.

Results Seventeen neonates were studied (gestation: 39.5[36-41] weeks; birth-weight: 3350[2590-4230]g). Hypoplastic left heart syndrome was the most common CHD. Milrinone was commenced at day $1-7$ (88\% on day 1$)$ of life, with heart rate $141 \pm[S D] 14 \mathrm{bpm}$, mean blood pressure $44 \pm 6 \mathrm{mmHg}$ and $\mathrm{CO} 479 \pm 147 \mathrm{ml} / \mathrm{min} / \mathrm{kg}$ at baseline. At $6 \mathrm{~h}, 24 \mathrm{~h}$ and $48 \mathrm{~h}, \mathrm{CO}$ was significantly increased by $23 \%, 20 \%$ and $28 \%$ from pre-treatment baseline, respectively, with increased anterior $(22 \%, 35 \%, 38 \%)$ and middle $(34 \%, 36 \%, 35 \%)$ cerebral $\mathrm{Vm}$. There were no significant changes in heart rate 
(150-160bpm), mean blood pressure $(43-46 \mathrm{mmHg})$ and RI during infusion. Plasma lactate concentrations decreased and urine output improved over $48 \mathrm{~h}$. One patient developed stroke pre-treatment; another had persistent hypotension after milrinone. Sixteen (94\%) neonates survived to hospital discharge.

Conclusions Milrinone improves CO and cerebral blood Vm without significant effects on blood pressure and RI. (Funded by Stollery Children's Hospital Foundation)

\section{THE REGIONAL HEMODYNAMIC EFFECTS OF DOXYCYCLINE IN NEWBORN PIGLETS WITH ASPHYXIA-REOXYGENATION}

doi:10.1136/archdischild-2012-302724.0320

IJ LaBossiere, 'JS Pelletier, 'D Bigam, 1,2PY Cheung. 'Surgery; ${ }^{2}$ Pediatrics and Pharmacology, University of Alberta, Edmonton, AB, Canada

Background Neonatal asphyxia has significant morbidities including hypoxic-ischemic encephalopathy, necrotizing enterocolitis and acute renal failure. These complications are associated with regional perfusion deficits. During reoxygenation, oxygen free radicals are produced and can activate matrix metalloproteinase-2, leading to cardiovascular dysfunction. There is little information regarding the effect of doxycycline, a known inhibitor of matrix metalloproteinase -2 , on regional perfusion. We hypothesized that doxycycline would improve regional hemodynamics during recovery in asphyxiated newborn piglets.

Methods Piglets (1-5 days old) were acutely instrumented for continuous monitoring of blood flow at the left common carotid, superior mesenteric and renal arteries (CAFI, SMAFI and RAFI, respectively). After stabilization, $2 \mathrm{hrs}$ of normocapnic alveolar hypoxia (10-15\% oxygen) was induced followed by 4 hrs of reoxygenation (21\% oxygen). Piglets were blindly randomized to receive either normal saline or doxycycline $(3,10$, or $30 \mathrm{mg} / \mathrm{kg}$ ) intravenously 5 minutes into reoxygenation ( $n=7 /$ group). Sham-operated piglets $(\mathrm{n}=5)$ received no hypoxia-reoxygenation.

Results All piglets had regional perfusion deficits at $2 \mathrm{hrs}$ of hypoxia (CAFI: $76 \pm[S D] 16 \%$; SMAFI: $57 \pm 21 \%$; RAFI: $11 \pm 10 \%$ of respective normoxic baseline). During 4 hrs of reoxygenation, doxycycline at $30 \mathrm{mg} / \mathrm{kg}$ increased RAFI $(\mathrm{p}<0.001)$ and CAFI $(\mathrm{p}=0.06$ ) (at 240min reoxygenation: $66 \pm 33 \%$ vs. $36 \pm 21 \% \quad[p=0.04]$ and $92 \pm 52 \%$ vs. $66 \pm 22 \%$ [p=0.09] for controls, respectively). Despite attenuating mesenteric hyperemia at $10 \mathrm{~min}$ of reoxygenation $(\mathrm{p}=0.03)$, there was no difference in SMAFI at the end of reoxygenation.

Conclusions In newborn piglets with hypoxia-reoxygenation, post-resuscitation administration of high-dose intravenous doxycycline improves carotid and renal hemodynamics and may attenuate transient mesenteric hyperemia during recovery.

Funded by Canadian Institutes of Health Research (CIHR)

\section{EARLY TARGETED CLOSURE OF PATENT DUCTUS ARTERIOSUS IN EXTREME PRETERM BABIES REDUCES BRONCHOPULMONARY DYSPLASIA}

doi:10.1136/archdischild-2012-302724.0321

'P Mallya, 'J Sundaram, 1,2S Gupta. 'Paediatrics and Neonatal Medicine, University Hospital of North Tees, Stockton; ${ }^{2}$ University of Durham, Durham, UK

Background PDA is common among very low birth weight babies. There is however growing controversy regarding treatment and clinicians are uncertain to treat or not to treat a PDA.

Objective To compare mortality and complications of prematurity in the following groups

1. Effect of gestation; babies $\leq 28$ weeks $\& 29-31$ weeks

2. Early $(<72 \mathrm{hrs})$ treatment vs. later symptomatic treatment of a PDA

3. Treatment vs. no treatment of a significant PDA
Methods All babies born < 32 weeks gestation were included in this study. A total of 223 babies met the inclusion criteria from January 2009 to September 2011. Of these 22 were excluded from analysis due to incomplete data. SPSS version 17(C) was used for data analysis.

Results

1. Babies $\leq 28$ weeks compared to 29-31weeks gestation had significantly higher PDA (61\%vs.23\%; p<0.05), Bronchopulmonary dysplasia (BPD) (40\%vs.12\%; $\mathrm{p}<0.05)$, duration of respiratory support (27days vs. 9 days; $p<0.05$ ) and mortality (16\%vs. $0 \%$; $<<0.05)$

2. Among babies with significant PDA, BPD was significantly reduced with early treatment of a PDA as compared to later symptomatic treatment (66.7\%vs.82.3\%; $\mathrm{p}<0.05$ )

3. There was significantly higher incidence of death among babies not treated for PDA as compared to those who received treatment ( $29 \%$ vs. $0 \%$; $p<0.001)$

\section{Conclusion}

1. Babies born $\leq 28$ weeks have higher burden of PDA, mortality and complications of prematurity.

2. Treatment of PDA significantly reduces mortality.

3. Early treatment of PDA significantly reduces BPD compared to later treatment.

\section{COULD CARDIAC ENZYMES AND THE CARINAL ANGLE MEASUREMENT BE USED AS INDICATORS OF HEMODYNAMICALLY SIGNIFICANT PATENT DUCTUS ARTERIOSUS?}

doi:10.1136/archdischild-2012-302724.0322

${ }^{1} \mathrm{E}$ Rahimov, ${ }^{2} \mathrm{Y}$ Ergul, ${ }^{3} \mathrm{Z}$ Ince, ${ }^{4} \mathrm{E}$ Yekeler, ${ }^{3} \mathrm{~S}$ Yildirim, ${ }^{5} \mathrm{~B}$ Omer, ${ }^{3} \mathrm{~A}$ Coban. ${ }^{1} \mathrm{C}$ Children's Health and Diseases; '2Pediatric Cardiology; ${ }^{3}$ Neonatology; ${ }^{4}$ Pediatric Radiology; ${ }^{5}$ Biochemistry, Istanbul University Istanbul Medical Faculty, Istanbul, Turkey

Aim In this blind prospective study, we aimed to examine the utility of cardiac enzymes and the carinal angle measurement in detecting hemodynamically significant patent ductus arteriosus (PDA), evaluating response to treatment and follow-up in preterm infants. Patients and Methods We evaluated 39 preterm infants $<32$ gestational weeks. Every patient had an echocardiogram (ECHO) taken on postnatal day 3 by a cardiologist blinded to clinical findings and laboratory results. ECHO was repeated when necessary. N-terminal pro-brain natriuretic peptide (NT-proBNP), cardiac troponin T $(\mathrm{c} \operatorname{Tn} \mathrm{T})$, creatine kinase $\mathrm{MB}$ isoenzyme (CK-MB) levels were measured on postnatal days 0,3 and 7 . The carinal angle on chest radiographs taken on the same days was measured. Those with PDA deemed hemodynamically significant based on ECHO were referred to as ehsPDA group. Those in this group who also had clinical signs were referred as sPDA subgroup.

Results The mean gestational age was 28.7 weeks; mean birth weight was $1120 \mathrm{~g}$. Initial ECHO showed PDA in 27 patients (69.2\%), 11 of which (40.7\%) had ehsPDA. Eight patients had sPDA When the ehsPDA and sPDA groups were compared to the rest, no statistically significant difference was found in terms of the carinal angle, NT-proBNP and cTnT. CK-MB levels were significantly low in ehsPDA and sPDA groups on postnatal days 3 and $7(p=0.017$, $\mathrm{p}=0.026$, respectively).

Conclusion NT-proBNP, cTnT, CK-MB and the carinal angle were not found useful in detection and follow-up of hemodynamically significant PDA.

323 ROLE OF AMINO TERMINAL PRO-BNP IN DIFFERENTIATING CARDIAC FROM RESPIRATORY PROBLEMS IN EGYPTIAN NEONATES PRESENTING WITH RESPIRATORY DISTRESS

doi:10.1136/archdischild-2012-302724.0323 